\title{
On the Radiation Mechanism of Pulsars
}

\author{
M. GREWING (Bonn) and H. HEINTZMANN (Köln)
}

\begin{abstract}
It is assumed that pulsars are rotating neutron stars surrounded by a strong dipolar magnetic field, the dipole axis being inclined with respect to the rotation axis. It is further assumed that the intense low frequency electromagnetic waves generated under such circumstances can indeed propagate. It can then be shown that charged particles interacting with such outgoing spherical waves are very efficiently accelerated to relativistic energies. During the acceleration which is almost instantaneous the particles emit a short duration pulse which resembles the observed emission characteristics of pulsars.
\end{abstract}

\section{Introduction}

Both from the great regularity and the characteristic time span between consecutive pulses as well as from the typical pulse duration and the observed fine-structure including the details of the polarization behaviour it has been inferred that pulsars are rotating magnetized neutron stars. Assuming the magnetic field to be of dipole form with its symmetry axis inclined with respect to the rotation axis of the star, it is necessary consequence of such a model that electromagnetic radiation is emitted the frequency of which is determined by the frequency of rotation of the star. In the case of the known pulsars this implies the generation of $0.3-30 \mathrm{~Hz}$ waves.

Due to their extremely low frequency and their large amplitude which is a consequence of the large surface magnetic fields expected for neutron stars from magnetic flux conservation arguments, the interaction of charged particles with such waves is a highly nonlinear phenomenon. The interaction can be characterized by parameter

$$
\mathrm{f}_{0}=\frac{\omega}{\Omega}=\frac{\mathrm{eB}}{\mathrm{mc} \Omega}
$$

where $\omega$ denotes the gyrofrequency of a particle of charge $e$ and mass $m$ in a magnetic field $B$, and $\Omega$ is the frequency of the wave. Inserting typical values for $B$ and $\Omega$ into equ. (1) where $B$ is determined either from the flux conservation argument $\left(B_{f}=B_{i}\left(R_{i} / R\right)^{2}, f=\right.$ final, $i=$ initial) or from the observed braking of the pulse period and $\Omega$ is directly taken from the observations, one finds that in all cases $f_{0} \gg 1$. This means that the gyroradius of the particle will be very mudh smaller than the wavelength. As first shown by GUNN and OSTRIKER (1969) the consequence of this is that charged particles caught by such waves are accelerated to relativistic energies immediately after coupling and that they are driven by the Lorentz-force $(v \times B, v \approx c)$ in the direction of the wave propagation.

\section{Equation of motion}

The general equation of motion for a charged particle moving in an electromagnetic field is given by

$$
\mathrm{mc} \mathrm{a}^{1}=\mathrm{mc} \frac{\mathrm{D} \mathrm{u}^{1}}{\mathrm{~d} \mathrm{~s}}=\frac{\mathrm{e}}{\mathrm{c}} \mathrm{F}^{\mathrm{k}} \mathrm{u}_{\mathrm{k}}
$$

where $D / d s$ denotes the covariant derivative along the path of the particle, $u^{1}$ its four-velocity and $F^{1 k}$ the electromagnetic field tensor. In equ. (2) we have neglected the radiation reaction term which is of minor importance for our considerations here (GREWING and HEINTZMANN 1971a). 
For spherical outgoing waves as generated by an oblique rotator the leading components of the electromagnetic field tensor are (DEUTSCH, 1955)

with

$$
\mathrm{rF}^{02}=\mathrm{E}_{\Theta}=\mathrm{B}_{\varphi}=\mathrm{rF}^{12}
$$

$$
\mathrm{E}_{\Theta}=\mathrm{B}_{\varphi}=\left\langle\mathrm{B}_{\mathrm{s}}\right\rangle \varrho_{\mathrm{s}}{ }^{3} \frac{\sin }{\underline{\varphi}} \frac{(\mu-\varphi-\varphi)}{\varrho}
$$

where we have introduced a spherical polar coordinate system $(r, \varphi, \Theta)$ and the dimensionless variables $\varrho=\Omega \mathrm{r} / \mathrm{c}$ and $\mu=\Omega \mathrm{t}$. $\left\langle\mathrm{B}_{3}\right\rangle$ denotes the average surface magnetic field of the star. Introducing as a further dimensionless variable $\xi=\Omega \mathrm{s} / \mathrm{c}$, and confining ourselves to particle motion near the equatorial plane $(\Theta=\pi / 2)$ the equations of motion read

$$
\begin{aligned}
& \gamma^{\prime}=\mathrm{f}_{0} \frac{\mathrm{f}(\mathrm{a})}{\varrho} \mathrm{u}_{\Theta} \\
& \mathrm{u}_{\varrho}^{\prime}=\frac{\left(\mathrm{u}_{\Theta}\right)^{2}}{\varrho}+\frac{\left(\mathrm{u}_{\varrho}\right)^{2}}{\varrho}+\mathrm{f}_{0} \frac{\mathrm{f}(\mathrm{a})}{\varrho} \mathrm{u}_{\Theta} \\
& \left(\varrho \mathrm{u}_{\varphi}\right)^{\prime}=\mathrm{o} \\
& \left(\varrho \mathrm{u}_{\Theta}\right)^{\prime}=\left(\gamma-\mathrm{u}_{\varrho}\right) \mathrm{f}_{0} \cdot \mathrm{f}(\mathrm{a})
\end{aligned}
$$

Here a dash denotes derivation with respect to $\xi$. We have introduced $\mathrm{f}_{0}=\left\langle\mathrm{B}_{\mathrm{s}}\right\rangle \varrho_{\mathrm{s}}{ }^{3} \mathrm{e} / \mathrm{mc} \Omega$ and $\mathrm{a}=\mu-\varrho-\varphi$, also $\gamma=\mathrm{d} \mu / \mathrm{d} \xi, \mathrm{u}_{\varrho}=\mathrm{d} \varrho / \mathrm{d} \xi, \mathrm{u}_{\Theta}=\varrho \mathrm{d} \Theta / \mathrm{d} \xi$ and $\mathrm{u}_{\varphi}=\varrho \sin \Theta \varphi / \mathrm{d} \xi$. Further we shall use $\alpha=\gamma-\mathfrak{u}_{\rho}$.

Equs. (5) have been solved by analytical as well as by numerical methods for a number of initial conditions (see e.g. GREWING and HEINTZMANN 1971a where also further references are given). In the following we consider the special case of particles caught in the wave-front of the spherical outgoing wave, i. e. particles coupling to the wave at essentially zero amplitude. The motion of the particle in this case is to a very good approximation given by (for details see GREWING and HEINTZMANN 1971a):

$$
\begin{aligned}
& a=a_{f}\left(1-\varrho_{0} / \varrho\right)^{1 / s} \\
& a=a_{0}\left(\varrho_{0} / \varrho\right)^{2} \\
& u_{\Theta}=\frac{\mathrm{f}_{0} a_{f}^{2}}{2 \varrho}\left(1-\varrho_{0} / \varrho\right)^{3 / s} \\
& \gamma_{Q} \approx u_{\varrho}=\frac{\mathrm{f}_{0}^{2} \mathrm{af}^{4}}{\varrho_{0}^{2}}\left(1-\varrho_{0} / \varrho\right)^{4 / 5} \\
& \varrho=\varrho_{0}+\Omega \mathrm{t}
\end{aligned}
$$

where $a_{i}=\left(40 \alpha_{0}^{2} \varrho_{0}^{3} / f_{0}^{2}\right)^{1 / 5}$ and quantities with a suffix nought denote values at particle injection time. This solution holds except for a very small time interval where $\varrho=\varrho_{0}$.

\section{Pulsar Radiation}

The almost instantaneous acceleration to relativistic energies of charged particles caught by intense low frequency electromagnetic waves that is shown by equs. (6) gives rise to a peculiar emission pattern. The flux emitted can be calculated from (cf. LANDAU and LIFSCHITZ 1967):

$$
\begin{aligned}
\mathrm{S}(\mathrm{t})= & -\frac{2 \mathrm{e}^{4}}{3 \mathrm{~m}^{2} \mathrm{c}^{3}} \mathrm{~F}_{\alpha \beta} \mathrm{u}^{\beta} \mathrm{F}^{\alpha} \gamma \mathrm{u}_{\gamma} \\
= & -\frac{2 \mathrm{e}^{4}}{3 \mathrm{~m}^{2} \mathrm{c}^{3}}\left(\gamma-\mathrm{u}_{Q}\right)^{2}\left(\varrho_{0} / \varrho\right)^{2} \mathrm{~B}_{\varphi}^{2} \\
& =\mathrm{S}_{0}\left(\Omega \mathrm{t} / \varrho_{0}\right)^{2 / s}\left(1+\Omega \mathrm{t} / \varrho_{0}\right)^{-32 / s}
\end{aligned}
$$

where we have used equs. (6) and introduced $S_{0} \approx\left(3 \mathrm{e}^{2 / c}\right) \times \Omega^{2} \alpha_{0}{ }^{1 / 15} \mathrm{f}_{0}{ }^{6 / 5} / \varrho^{4 / 5}$. 


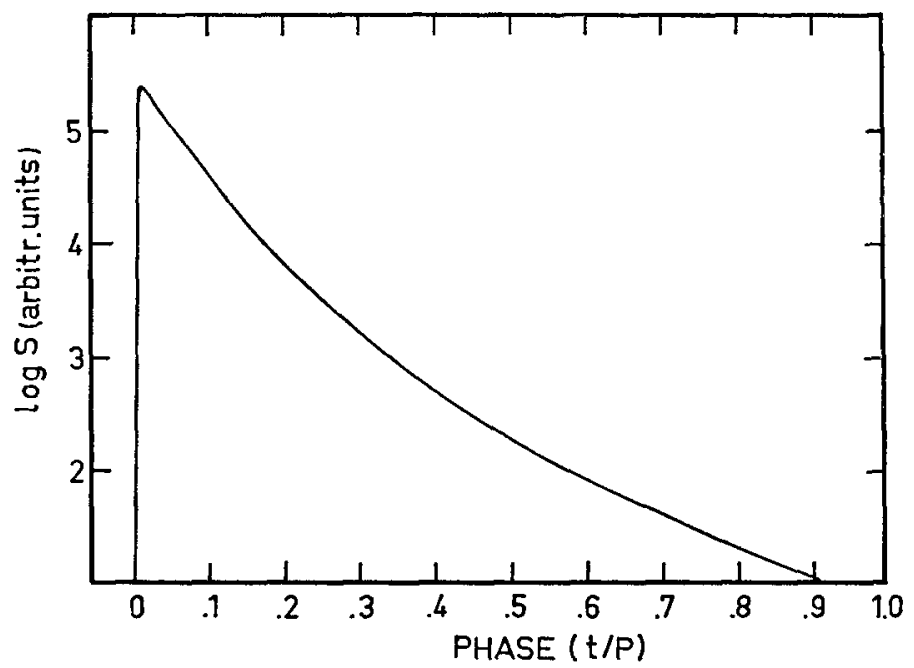

Fig. 1: The flux emitted by a charged particle coupling to an intense low frequency wave at zero amplitude $(\mathrm{P}=2 \pi / \Omega$.

From equ. (7) it is immediately evident that the emitted flux is confined to a single sharp pulse (see also Fig. 1) the duration of which is W/P $=0.05 \varrho_{0}$ independent of the particular initial conditions ( $W=$ pulse width at half the maximum intensity, $P=$ wave period). Assuming $\varrho_{0}=1$, i. e. coupling to the wave at the "velocity of light cylinder", and taking a typical pulsar period of $1 \mathrm{sec}$, one would expect a typical pulse duration of $50 \mathrm{msec}$. This is in basic agreement with the observations and might indicate that the peculiar radiation characteristics of pulsars are indeed linked to the characteristic emission pattern of charged particles coupling to the intense low frequency waves that rotating magnetized neutron stars should generate. However, both the total energy output and the frequency spectrum remain to be explained. Choosing particular initial conditions GREWING and HEINTZMANN (1.971b) were able to fit a model to the optical and X-ray emission of the Crab pulsar. But much more detailed calculations including further components of the electromagnetic field in the vicinity of a rotating dipole are needed until such models can be generalized.

$$
\text { Refere nces : }
$$

DEUTSCH, A. J., 1955, Ann. d'Aphysique 18, 1.

GREWING, M., Ig71a, Astron. Astrophys. (in press).

HEINTZMANN, H., 1971b, Astrophys. Lett. 8, 167.

GREWING, M., and HEINTZMANN, H., 1971a, Astron. Astrophys. (in press).

GREWING, M., and HEINTZMANN, H., 1971b, Astrophys. Lett. 8, 167.

GUNN, J. E., OSTRIKER, J. P., 1969, Phys. Rev. Lett. 22, 728.

LANDAU, L. D., LIFSCHITZ, E. M., 1967, „Klassische Feldtheorie“ (Akademie-Verlag, Berlin).

OSTRIKER, J. P., GUNN, J. E., 1969, Astrophys. J. 157, 1395. 